Int. J. Dev. Biol. 49: 479-489 (2005)

doi: $10.1387 / \mathrm{ijdb} .041929 \mathrm{ys}$

\title{
Plant stem cell niches
}

\author{
YVONNE STAHL and RÜDIGER SIMON* \\ Institut für Genetik, Heinrich-Heine Universität Düsseldorf, Düsseldorf, Germany
}

\begin{abstract}
Stem cells are required to support the indeterminate growth style of plants. Meristems are a plant's stem cell niches that foster stem cell survival and the production of descendants destined for differentiation. In shoot meristems, stem cell fate is decided at the populational level. The size of the stem cell domain at the meristem tip depends on signals that are exchanged with cells of the organizing centre underneath. In root meristems, individual stem cells are controlled by direct interaction with cells of the quiescent centre that lie in the immediate neighbourhood. Analysis of the interactions and signaling processes in the stem cell niches has delivered some insights into the molecules that are involved and revealed that the two major niches for plant stem cells are more similar than anticipated.
\end{abstract}

KEY WORDS: stem cells, initials, root meristem, shoot meristem

\section{Stem cell primer}

\section{What are stem cells?}

Stem cells are cells capable of unlimited self-renewal that have the potential to generate differentiated descendants. Differentiation can be defined as qualitative changes in the cellular phenotype that are caused by changes in gene expression that ultimately lead to functional competence. Most of the current discussions about the qualities and characteristics of stem cells stems from research on animals. There, stem cells are found at different developmental stages and in a variety of tissues. For example, there are hemopoietic stem cells residing in the bone marrow that can produce a range of different blood cells, or epidermal stem cells that can regenerate skin after injuries. Such stem cells are specialised for the production and regeneration of specific cell types. With this restricted potential to generate one or more highly defined types of offspring, they are described as multi- or pluripotent. The only truly totipotent animal stem cells that can form all cell types of the body are embryonic stem cells, or the zygote. During ontogeny, the daughters of these initially totipotent stem cells become gradually restricted and differentiate into the stem cells of adult tissues.

Do plants have bona fide stem cells? This question was controversial, since protoplasting and in vitro culture allows regeneration of embryos or whole plantlets from differentiated leaf cells of many plant species. This would suggest that all leaf cells can act as stem cells - or that the stem cell concept cannot be applied to plants. However, the traditional view of animal stem cell potential has been recently challenged with the discovery that stem cell fates in adults are still plastic and can be altered by cues from the environment (Blau et al., 2001). The evolving view that emerged from numerous studies is that stem cells are not necessarily specific cellular entities, but that they represent a function that can be assumed by numerous cell types. In the most extreme view, all cells receiving the correct signal (or a combination of signals) will then adopt the stem cell state. Thus, leaf cells that are protoplasted and grown in tissue culture will be exposed to a variety of new signals such as plant hormones or compounds released into the medium and these signals can trigger the fate switch to an undifferentiated, stem cell-like state that allows to regrow an entire plant. Indeed, a receptor-kinase perceiving an as yet unidentified signal has been identified that promotes embryogenic competence of cultured Arabidopsis cells (Hecht et al., 2001). So the hidden totipotency of many plant cells that can be revealed in tissue culture appears less alien when we accept the modern view of stem cell identity as a transient cellular state that is controlled by appropriate environmental cues.

\section{Where do they thrive?}

Noting that local regulators direct a stem cell's fate puts extra emphasis on the microenvironment, or niche, where stem cells reside (Watt and Hogan, 2000). Stem cell descendants that become evicted from these safe havens may face terminal differentiation or death. Identifying these niches and finding the stem cells within has not been a trivial task in animal systems: with a few exceptions, such as the Drosophila gonads and peripheral nervous system, where stem cells have a defined orientation relative to surrounding cells, molecular markers are needed to identify stem cells in most tissues. A plant's stem cell niches are the meristems and since plant cells are immobile, stem cells are

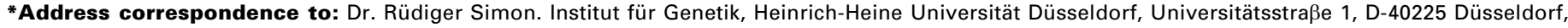
Germany. Fax.: +49-211-811-2279. e-mail: ruediger.simon@uni-duesseldorf.de
} 
readily identified by their position within meristems. There are several types of meristems: The shoot, root and vascular meristems all carry their separate and specialized set of stem cells.

\section{How do they work?}

Although stem cells have a high capacity for self renewal and are essential for tissue production and/or regeneration, they divide only infrequently. In most cases, stem cell descendants do not directly differentiate, but form an intermediate cell population of more rapidly dividing committed progenitors. These transit amplifying cells (TA cells) have a limited proliferative capacity and an already restricted differentiation potential. Their main task is to increase the population of cells generated from a single stem cell division.

Because stem cell divisions result in two different types of progeny, they are intrinsically asymmetric. However, there are at least two general strategies how this asymmetric outcome can be achieved. In the case of invariant or divisional asymmetry, stem cell divisions produce strictly one daughter that remains as a stem cell, the other daughter differentiates or aquires TA cell fate (Fig. 1). The second option is populational or environmental asymmetry: after division, stem cell daughters have a finite probability of being either stem cells or committed progenitors that follow a path to differentiation (Fig. 2). Although individual divisions may produce two stem cells or two differentiating daughters, the system is balanced at steady state and produces on average one stem cell and one differentiating cell per division. The differences between these two strategies lie mainly in the mechanisms of regulation: Divisional asymmetry can involve an asymmetric partitioning of cell fate determinants, or the displacement of a daughter from the stem cell niche. Populational asymmetry depends on extrinsic controls that sense and communicate the size of the stem cell population and regulates the probability of stem cell daughters to maintain stem cell fate. Both strategies involve extensive communication between stem cells and their niches. We will discuss in this review if and how these alternative strategies may be employed by plant stem cells and what we know so far about the molecules involved.

\section{Deconstructing plant stem cell niches: shoot mer- istems}

The shoot meristem gives rise to the above-ground organs of a plant and produces the main stem. At its flanks, new specialized meristems can be initiated that produce flowers. Both types of meristems are structurally very similar. Cells in these meristems are arranged in layers and zones.

\section{Layers}

Sections through the shoot meristem centre along the apical-basal axis reveal an organisation into discrete cell layers (Fig. 3). The outermost cell layer (L1) consists of epidermal progenitor cells, the next cell layer (L2) will gives rise to subepidermal tissues and also to the gametes. Cells underneath produce the majority of the plant's ground tissue; although they are not arranged in a single sheath like layer, they are called layer 3 (L3). L1, L2 and L3 represent cell clones that are maintained during development. This is possible because cell divisions in L1 and L2 are strictly anticlinal (cell walls perpendicular to surface) and daughter cells therefore stay in their layer of origin. In the L3, cells can divide in all directions, but cells generally do not invade the adjacent L2. Such a strict separation of layers in the entire meristems necessitates stem cell activity in each individual layer. This layered organisation is found in most angiosperm shoot meristems, but its functional relevance is unclear. However, a recent study by Reinhardt and colleagues (Reinhardt et al., 2003) showed that removing only the L1 layer by laser ablation affects the orientation of cell divisions in the L2. These now shift from their normal anticlinal pattern to periclinal, causing local tissue outgrowth. So one role of the $L 1$ is to control division patterns in deeper meristem regions. Furthermore, meristems arrested development when the entire $L 1$ was removed, suggesting that the $L 1$ also provides an essential function or signal for meristem maintenance.

\section{Zones}

Organ primordia that develop into leaves or flowers originate from the meristem flank or peripheral zone of the shoot meristem. Stem cells are located in the central zone of the meristem, which is surrounded by the peripheral zone. Cells in a deeper region of the meristem form the rib zone that gives rise to the majority of the ground tissue in the plant body. Chimeras have been used to identify the fate of descendants from the three zones. In sectorial chimeras, only a part of the plant is marked with a cell clone. The size of this clone or sector along the apical-basal axis indicates for how long the progenitor cell has resided in the shoot meristem; sectors that extend from the plant's base to its tip indicate that a stem cell was marked. Such clonal analysis has been used to conclude that at least 3 to 4 stem cells are present in each meristem layer that together form the central zone of the meristem (Stewart and Dermen, 1970). Maintenance of this stem cell pool in the central zone is

Fig. 1. Divisional or invariant asymmetry. The outcome of a stem cell division is controlled through signal exchange between the stem cell and the surrounding niche cells. Stem cell daughters undergo several rounds of cell divisions as a trasit amplifying cell (TA cell) before differentiation. Stem cell promoting signals in yellow, feedback signals in red.

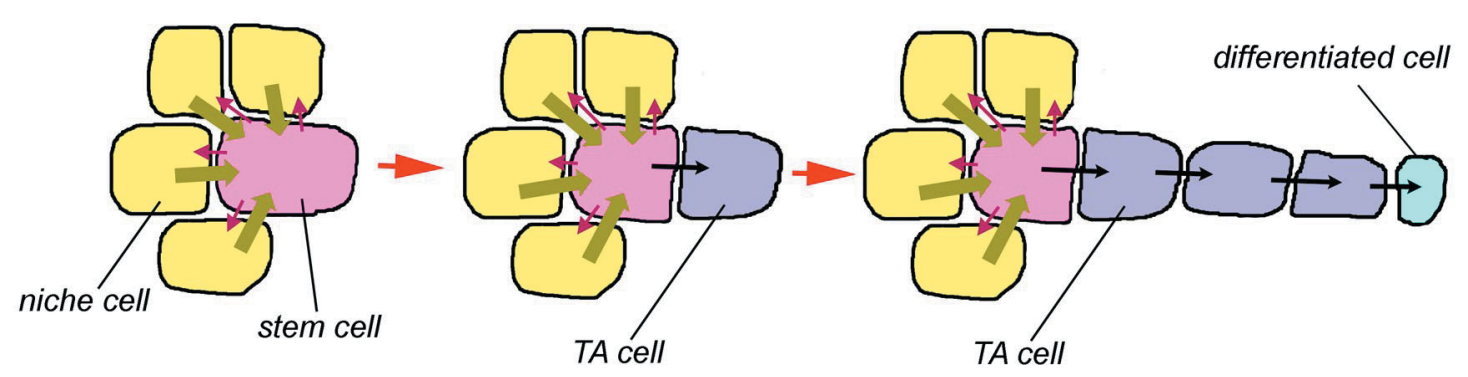


essential to allow indeterminate growth of the shoot apical meristem.

\section{Cell division rates, microsurgery and impermanence of the stem cell state}

There is an obvious division of labour between the central zone harbouring stem cells and the peripheral zone as site of organ initiation. This is also reflected in the cell division rates that vary vastly across the SAM surface. Live imaging of Arabidopsis shoot meristems revealed that rates are generally higher in the peripheral zone than in the centre. Cell cycle lengths range from 36-72 $\mathrm{h}$ in the central zone to 12-36 $\mathrm{h}$ in the peripheral zone and young organ primordia (Reddy et al., 2004). Thus, when a stem cell daughter leaves the central zone, it will soon change its behaviour and start to divide more rapidly, consistent with the concept of TA cells increasing the population size of stem cell descendants. In contrast to the root meristem, where a group of non-dividing cells constitute the quiescent centre (see below), all cells of the shoot meristem divide regularly. When and where a cell divides appears to be influenced by its neighbours, since small groups of cells were found to divide coordinately within a short time window. This coordinated behaviour was observed among cells within the same cell layer, but also between cells in adjacent layers that therefore belong to different clones (Reddy et al., 2004). Laser induced cell ablation technology has offered a dramatic way of revealing such local interactions. For example, when the entire central zone of a tomato meristem was removed by laser pulses, a new central zone initiated from the peripheral zone within 4 days after the treatment (Reinhardt et al., 2003). This new zone harboured functional stem cells that were able to support further growth. In a normal (untreated) shoot meristem, neighbouring cells must then be inhibited from acquiring stem cell or niche fate by the presence of a functional central zone. The mechanism of this proposed inhibition is so far unknown. However, the results from these precise cell ablation experiments highlight again two important facts about stem cell biology: (1) the current state of a cell (stem cell or non-stem cell) is not permanently fixed, but flexible; and (2), the stem cell state is controlled by interactions with the immediate cellular environment, the stem cell niche.

\section{Organisation of the stem cell niche}

Longevity and continuous activity of the shoot meristem is only possible if a pool of stem cells is maintained that will replenish and restore the meristem cell pool, since cells are constantly lost from the meristem when organs are formed at the periphery. Shoot meristem stem cells were first identified as cells that divide infrequently and it was occasionally questioned that they divide at all. In the last 10 years, we have started to understand stem cell behaviour in shoot meristems. Most of the information was collected from studies with Arabidopsis, but related genes and mechanisms governing stem cell development have been identified in other plants species such as maize. Stem cell number in the shoot apical meristem of Arabidopsis is controlled by (at least) two antagonistic pathways that promote or repress stem cell activity, respectively. These two pathways involve the exchange of signals along the apical-basal axis of the plant; between stem cells at the meristem tip and a group of cells in the deeper L3 layer that form the organizing centre $(\mathrm{OC})$ (Figs. 3,4). A key element of the stem cell promoting pathway is the WUSCHEL (WUS) gene, encoding a homeodomain transcription factor (Laux et al., 1996, Mayer et al., 1998). In the absence of WUS function, meristems are initiated, but they fail to keep a sufficient number of stem cells to maintain prolonged growth and development. WUS is first expressed at the 16-cell stage of embryogenesis in cells that will form the shoot meristem and later on, WUS RNA is found only in the $\mathrm{OC}$ cells that lie underneath the stem cells. Therefore, WUS has to act non-cellautonomously to promote stem cell fate at the meristem tip. So far, there is no indication that the WUS protein itself is moving out to adjacent cells (which has been observed for other plant transcription factors), but WUS may generate the signal that promotes stem cell identity in the neigbourhood. Why stem cell fate is only induced in the cells apical to the $\mathrm{OC}$ is not known.

\section{CLV signaling regulates WUS activity}

Mutant hunts identified additional genes controlling stem cell fate. Mutations in one of the three CLAVATA genes ( $C L V 1,2$ and 3) cause stem cell accumulation in shoot and floral meristems. $C / V$ mutant meristems maintain more stem cells in the central zone than required to support normal meristem growth, resulting in enlarged meristems that occasionally lose any growth restriction and start to fasciate. This size increase of the central zone has also direct consequences for organ number: the peripheral zone that surrounds the meristem centre enlarges correspondingly, allowing the formation of more organs. In normal development, the $C L V$ genes act together and restrict stem proliferation. CLV1 encodes a receptor kinase, consisting of extracellular leucinerich-repeats, a transmembrane domain and a cytoplasmic serine/ threonine kinase domain (Clark et al., 1997). CLV1 is expressed in the meristem center, enclosing the domain of WUS expression. The CLV2 protein is a LRR-receptor that lacks the kinase moiety (Jeong et al., 1999) and CLV1 may form a heterodimeric receptor with CLV2 to form the core of a larger receptor complex (Trotochaud et al., 1999). The size increase of $c / v 1$ mutant meristems shows that the $C L V$ signaling pathway restricts stem cell fate (or



Fig. 2. Populational or environmental asymmetry. Wether a stem cell division produces the same or different daughter cell types depends again on niche cells. Stem cells may divide asymmetrically to produce TA cells (bottom row) or give rise to new stem cells (top row). The differentiating cell population constantly signals back to the niche and stem cells to readjust the outcome of stem cell divisions. 
promotes differentiation), acting antagonistically to WUS. Indeed, WUS is expressed in a larger domain in $c / V$ mutants, indicating that WUS is negatively regulated by the $C L V$ genes. The third CLAVATA gene, CLV3, encodes a small secreted protein that is expressed only in stem cells (Brand etal., 2000, Fletcher etal., 1999, Rojo et al., 2002). Current evidence supports a model whereby CLV3 is secreted from stem cells and activates CLV1 signaling in cells underneath to repress WUS expression (Brand et al., 2000). Since CLV3 is expressed in the stem cells of the shoot system, it depends again on WUS expression. This mutual regulation and interdependence between $C L V 3$ and WUS can then establish a feedback regulated circuitry: if stem cell number increases, the amount of CLV3 protein will increase correspondingly, causing a downregulation of WUS via CLV1 activation (Brand et al., 2000, Schoof et al., 2000) (Fig. 4A). This lowered amount of WUS (smaller stem cell niche) allows only fewer stem cells to be maintained. Fewer stem cells will provide a weaker negative feedback signal (CLV3), allowing WUS expression to increase again. High WUS expression (larger stem cell niche) will induce supernumerary stem cells, which will again be counteracted by a concomitant increase in $C L V$ signaling and WUS repression. This circuitry of stem cell regulation remains continously active. Shoot and floral meristems therefore use the strategy of populational asymmetry for stem cell deployment. The probability of a stem cell daughter to remain a stem cell then depends on its position in the meristem: a cell that is displaced laterally after a division will receive less (or no) WUS derived signal and adopt TA cell fate.

\section{Stem cell regulation beyond Arabidopsis}

The components of the CLVIWUS stem cell regulator appear to be used in many plant species. In petunia, TERMINATOR (TER) represents the orthologue to WUS of Arabidopsis and ter mutants terminate shoot meristem activity at early stages (Stuurman et al., 2002). Mutations in the FASCIATED EAR2 ( $F E A 2$ ) gene of maize cause a massive overproliferation of the inflorescence meristem; the FEA2 protein localises to the plasma membrane and shows high sequence similarity to CLV2 of Arabidopsis (Taguchi-Shiobara et al., 2001). In rice, a CLV1 orthologue has been identified as FLORAL ORGAN NUMBER1 (FON1). Mutations in fon 1 cause proliferation of meristem cells and initiation of additional floral organs (Suzaki et al., 2004). In contrast to CLV1 of Arabidopsis, which is expressed only in L3 layer, FON1 RNA is present throughout floral meristems. Finally, chimeras that were generated by grafting between wildtype and fasciated (fas) mutant tomatoes had shown that meristem size in tomato (like in Arabidopsis) is controlled from the L3 layer (Szymkowiak and Sussex, 1992). The gene product of the tomato FAS gene is not yet known, but in analogy to Arabidopsis, it may encode a component of a $C L V$ signaling pathway that controls expression of a tomato WUS-orthologue in the L3.

\section{Modulation of $C L V$ signaling}

The model for stem cell regulation in Arabidopsis by $C L V /$ WUS allows to keep the number of CLV3 expressing stem cells proportional to the number of WUS expressing cells underneath; however, it is not sufficient to account for homeostasis of absolute stem cell number. Obviously, meristem size may change during development, although only within a narrow range. For example, a seasonal increase in organ production rates may require that more stem cells are maintained in the meristem. The activity of the CLVIWUS stem cell regulator has to be flexible to allow for such moderate changes, but also robust enough to guarantee maintenance of an appropriate stem cell number during changing environmental conditions.

Several factors have been identified that modulate $C L V$ signaling: one negative regulator of $C L V$ signaling is KAPP, a protein phosphatase that interacts with a variety of receptor kinases 
(Stone et al., 1998, Williams et al., 1997). Another candidate regulator is the POLTERGEIST (POL) protein phosphatase $2 \mathrm{C}$; pol mutants have been identified as phenotypic suppressors of hypomorphic $c / v 1$ mutations, indicating that $P O L$ activity interferes with $C L V$ signaling (Yu et al., 2003, Yu et al., 2000). Similar to $K A P P, P O L$ is not only expressed in meristems but in many plant tissues, indicating that $P O L$ may function in several signal transduction pathways (Interestingly, a recent analysis of the pol mutant phenotypes suggested that $C L V$ signaling may act upon additional, but as yet unidentified targets besides WUS (Yu et al., 2003)). Phosphatases like POL and KAPP could act to dampen any (accidental) dramatic increase in $C L V$ signaling that could switch off WUS expression and cause a terminal loss of meristem stem cells. SHEPHERD ( $S H D$ ) affects $C L V$ activity at a very different level (Ishiguro et al., 2002). The $S H D$ gene encodes an ER-resident HSP90-like protein that is required for the proper folding of CLV proteins. Homozygous shd mutants form larger shoot and floral meristems, thus resembling $c / v$ mutants, but they also show defects in root meristem organization and pollen tube growth. It is likely that SHD acts in the assembly of several receptor signaling complexes and does not have an exclusive role in the regulation of the $C L V$ pathway.

\section{Specificity of the CLV signaling components}

There are at least 610 receptor-like kinases encoded in the Arabidopsis genome, many of them showing high amino acid sequence similarity with CLV1 and some of the about 220 transmembrane LRR receptors could have partially overlapping functions with CLV1 in meristem regulation. This is supported by the recent observation that all previously described $c / v 1$ alleles that exhibit strong or intermediate phenotypes are dominant-negative alleles (Dievart et al., 2003); c/v1 null alleles result in only weak phenotypes and are clearly distinguishable from null mutants in CL V3. Thus, CLV3 may signal via CLV1 and additional related receptors that have not been identified so far. The mutant clv1 protein encoded by strong $c / v 1$ alleles is then predicted to bind to and inhibit the activation of these redundant receptors.

The CLV2 protein is not necessarily involved in a specific interaction with the CLV3 ligand, but may rather promote the stability of the CLV1 receptor kinase within the complex. Stem cells accumulate in $\mathrm{clv} 2$ mutants because the amount of CLV1 receptor available for signal transmission is reduced. However, the $c / v 2$ floral phenotype is suppressed when plants are grown under short day conditions, i.e. CLV2 is only required under long days. This could suggest that other receptor proteins can substitute for CLV2 under certain conditions (Jeong et al., 1999, Kayes and Clark, 1998). Jeong et al. proposed that long photoperiod repress the activation of an unknown receptor kinase that acts independently of the $C L V$ pathway to restrict WUS activity (Jeong and Clark, 2004). All c/v2 mutants display relatively weak meristem phe- notypes compared to c/v1 or c/v3 mutants, but show also defects in organ development such as pedicel elongation. Consistent with additional functions outside the meristem, $C L V 2$ is expressed throughout the plant.

$C L V 3$ was first described as a unique sequence; meanwhile, more than 40 genes have been identified in the Arabidopsis and other plants' genomes that encode CLV3-like (CLE) proteins (Cock and McCormick, 2001). The sequence similarities between them are confined to an N-terminal secretion signal and the CLEmotif, a short stretch of 14 amino acids near the $\mathrm{C}$-terminus. Both c/v3-1 and c/v3-5 mutant alleles carry a point mutation in this region, indicating that the CLE motif is required for protein function (Fletcher etal., 1999). Of the 25 Arabidopsis CLE-genes (including $C L V 3$ ), 17 are expressed at detectable levels in the shoot apex (Sharma et al., 2003). Although detailed studies have still to be perfomed, we can assume that several CLE proteins are also expressed in the meristem, available for binding to the CLV receptor complex. Therefore, the recognition of CLV3 by CLV1 must be highly specific to avoid cross-signaling by other CLEproteins; alternatively, a low binding specificity can be tolerated if only CLV3 is expressed at sufficient levels in the meristem to interact with CLV1. Indeed, misexpression of CLE4O from the $C L V 3$ promoter rescued the meristem defects of $c / V 3$ mutant plants, indicating that CLV3 is singular in its expression pattern, but not in its capacity to interact with CLV1 (Hobe etal., 2003). We will give more attention to the roles of CLE proteins in the discussion on root stem cells.

\section{Is WUS sufficient to make stem cells?}

The loss of stem cells in wus mutants tells us that WUS is required for stem cell maintenance. However, is WUS expression alone also sufficient to induce stem cell fate in just any given cell? This has been tested by misexpressing WUS using a variety of different promoters. WUS was shown to induce the expression of the stem cell marker $C L V 3$ even in differentiating organs (Schoof et al., 2000), sugesting that WUS alone can induce stem cells. However, not all cells were able to respond to increased WUS activity, indicating that a specific cellular competence (or additional signals from the microenvironment) is required. For ex-
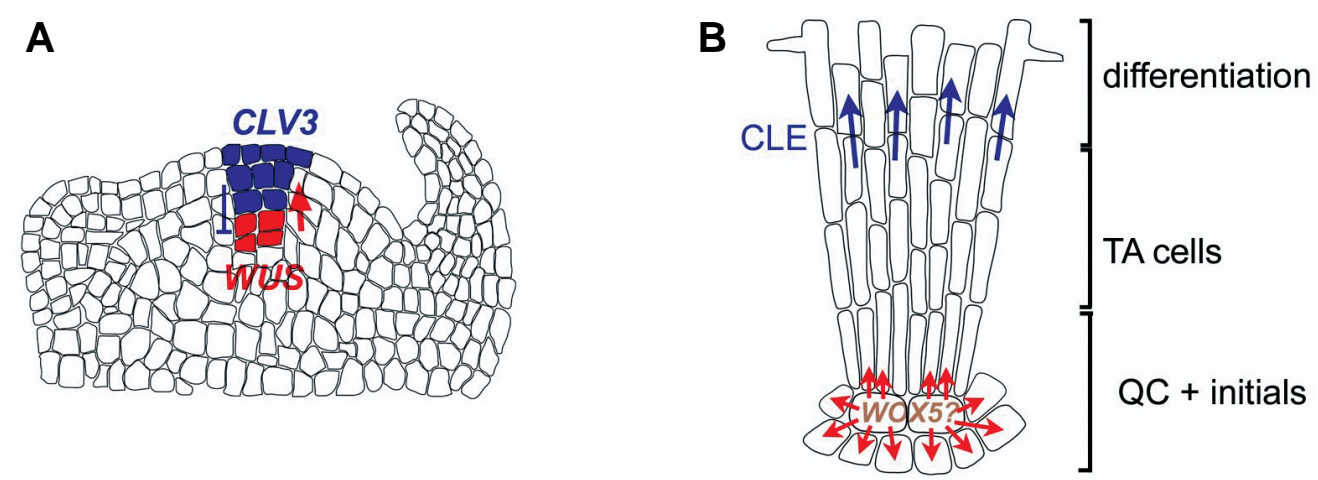

Fig. 4. Possible CLV signaling pathways in shoot and root meristems. (A) In shoot and floral meristems, WUS acts from the organising centre (OC) to induce CLV3-expressing stem cells in the central zone. The stem cell population signals back via the CLV pathway to restrict WUS expression. (B) In the root, quiescent centre (OC) cells signal (red arrow) to adjacent cells to maintain their status as initials. A WUS-related gene (WOX5) is expressed in the $Q C$ and could be involved in this signaling process. Activation of CLE expression promotes early differentiation of TA cells, possibly via a CLV-like signal transduction pathway. 
ample, WUS expression in leaves was reported to activate $C L V 3$ expression only in (adaxial) leaf axils (Brand et al., 2002) or in the vasculature (Lenhard et al., 2002), but not in all leaf cells. The adaxial sides of the leaf base are regions that can give rise to new axillary meristems later in development and the vasculature contains its own meristematic cells that may be responsive to ectopic WUS activity. Interestingly, high level WUS expression in roots was reported to cause the de-novo formation of embryos from root cells, suggesting that WUS may have additional and previously unsuspected roles in embryogenesis (Zuo etal., 2002). Alternatively, the root context could affect the interpretation of the WUS signal. Gallois and colleagues further refined these experiments by using a heat-shock inducible recombination system to activate high-level WUS expression in random root cells (Gallois et al., 2004). WUS expression in the root tips was able to activate the CLV3 (shoot) stem cell marker within 3 days after induction. Like in the shoot meristem, $C L V 3$ and WUS were not expressed in the same, but mostly in adjacent cells. In the following weeks, these root tips formed green leaf tissue, indicating that root cells can be reprogrammed to shoot stem cell identity and that WUS expression can trigger this transformation. However, in combination with external auxin, WUS expressing root tips generated entire embryos and together with the floral regulatory protein LEAFY, flower organs were initiated. Thus, the specific response of a cell to WUS is dependent on the environment. Such a context dependent interpretation of the WUS signal is also seen during flower development. In floral meristems, WUS activates expression of the homeotic gene AGAMOUS ( $A G$, discussed below) (Lenhard et al., 2001, Lohmann et al., 2001). Later in ovule development, WUS is expressed in the nucellus and promotes the initiation of the integuments from the chalazal region (GrossHardt et al., 2002). Taken together, WUS does not appear to provide a signal that is specific for the generation of only stem cells. Instead, the specific response of a cell to a WUS derived signal may depend entirely on a cell's microenvironment or developmental history. There are several WUS -related genes encoded in the Arabidopsis genome that play a role in embryonic pattern formation, development of the lateral axis and possibly specification of the root meristem's quiescent centre (Haecker et al., 2004, Matsumoto and Okada, 2001). The WUS gene family, together with their immediate target genes, could form signaling modules that are used repeatedly during plant development to mediate communication and coordinated growth between adjacent cell groups.

\section{Terminating stem cells in flower meristems}

In floral meristems, stem cells are maintained only for a limited period, i.e. as long as new organs are formed. They are then consumed during the production of the innermost floral organs, the carpels. Like in the shoot meristem, the $C L V I W U S$ circuitry controls stem cell fate also in flowers: wus mutant flowers form fewer stamens and lack the central carpels, indicating that stem cells were consumed prematurely; $c / v$ mutants accumulate stem cells in floral meristems that are then used to form additional carpels or even extra floral whorls, resulting in a misshapen, clublike gynoecium (hence the name CLAVATA, derived from clava (lat.) = club). However, there are also other regulators acting exclusively in floral meristems. One of them is AGAMOUS $(A G)$, encoding a MADS box transcription factor that controls the production of stamens and carpels in the inner two flower whorls (Yanofsky et al., 1990). The floral meristems of ag mutants become indeterminate, implicating $A G$ in the restriction of stem cell number. In wildtype floral meristems, WUS expression is lost around floral stage 6, but WUS remains expressed in ag mutant flowers beyond that stage (Lenhard et al., 2001, Lohmann et al., 2001). Furthermore, WUS activity is required for the indeterminacy of ag mutant flowers, indicated by the lack of floral organ proliferation in ag/wus double mutants (Laux et al., 1996). Thus, $A G$ appears to control meristem stem cell proliferation by negatively regulating $W U S$ expression. How $A G$ performs this function is still unknown; however, it appears that $A G$ acts independently of the $C L V$ genes, since floral meristem indeterminacy is further enhanced in $a g / c / v$ double mutants and the WUS expression domain expands further in $a g / c / v$ mutant meristems compared to those of ag or $c / v$ single mutants (Lohmann et al., 2001).

This role of $A G$ in the control of meristem determinacy is restricted to flowers. In the shoot meristem, $A G$ expression is not activated. One important factor that distinguishes floral meristems from shoot meristems is the expression of LFY protein. LFY is a transcription factor that can convert shoot to flower meristems by activating a set of flower specific regulator genes (Parcy et al., 1998, Weigel et al., 1992). Activation of $A G$ in the centre of floral meristems is controlled by the combined activities of LFY, which is expressed throughout floral meristems and WUS. First evidence for this role of WUS in the activation of a floral homeotic regulator gene came from misexpression experiments, where WUS transcription was controlled from promoters that direct organ specific gene expression in flowers (Lenhard et al., 2001, Lohmann et al., 2001). For example, expression of WUS from the $A P 3$ promoter (in the presumptive second and third floral whorl) resulted in the production of extra floral organs that developed into carpelloid stamens - both organ types require $A G$ activity. Indeed, increased WUS expression caused proliferation of meristem cells and ectopic activation of $A G$ expression. A more detailed analysis at the molecular level then showed that the WUS protein can bind specific DNA target sequences of the $A G$ regulatory regions that are located immediately adjacent to the binding sites for the LFY protein (Lohmann et al., 2001). Both proteins can act independently to activate $A G$ expression in a yeast assay system. However, since $A G$ is normally only expressed in the centre of floral meristems, both $\angle F Y$ and WUS appear to be required for normal levels of $A G$ transcription.

From stage 3 until stage 6, both $A G$ and $W U S$ are expressed in an overlapping set of cells, indicating that $A G$ expression per se is not sufficient to downregulate WUS transcription. Furthermore, ectopic expression of $A G$ throughout the plant causes early flowering and homeotic organ transformations, but does not terminate shoot meristem development (Mizukami and Ma, 1992). This indicates that either $A G$ expression levels have to reach a certain threshold level, or accumulation of additional and so far unidentified factors is required to cooperate with $A G$ in the regulation of $W U S$ and consequently meristem determinacy. The precise timepoint during development that this happens may be of great importance, since a premature loss of stem cells could result in sterile flowers if carpels are not formed. We may therefore expect that $A G$ activity is tightly controlled and possibly at several levels. Indeed, in addition to positive and negative regulation of $A G$ transcription, there is also posttranscriptional control of $A G$ 
activity. Several of the factors involved were first identified through sensitized genetic screens, i.e. by searching for extragenic mutations that enhance the meristem indeterminacy phenotype of a weak ag mutant (Chen and Meyerowitz, 1999). At least 4 genes (HUA1 and 2, HEN2 and 4) have so far been shown to affect the generation of functional $A G$ mRNA (Chen et al., 2002, Cheng et al., 2003, Li et al., 2001, Western et al., 2002). At the molecular level, HUA1,2 and HEN4 seem to promote the processing of (or inhibit aberrant polyadenylation within) the unusually large second intron of $A G$. Interestingly, it is this second intron which also contains the key sequences for both positive and negative regulation of $A G$ transcription (Busch et al., 1999, Sieburth and Meyerowitz, 1997). An additional member of the $A G$ dependent floral termination pathway, $H E N 3$, was recently shown to encode a cyclin dependent kinase. Repression of WUS at later stages of flower development is alleviated in hen3 mutants, providing a potential link between stem cell maintenance and cellular proliferation (Wang and Chen, 2004).

From what we have discussed so far, a picture of stem cell control in meristem emerges: When meristems are first formed, WUS is activated in a small set of cells and signals to specifiy stem cells at the meristem tip. These stem cells in turn express CLV3, which acts via the CLV1/2 receptor complex to restrict WUS expression. After floral induction, floral meristems are formed at the flanks of the shoot apical meristem that express the LFY transcription factor. The combined activity of LFY and WUS induces transcription of $A G$ in the central zone of the floral meristems. AG now acts at two levels: it specifies the production of stamens and carpels, in combination with several other genes and switches off WUS expression. Thus, WUS is controlled by two and probably independently acting feedback regulated systems. The determinate state of floral meristems, with a complete

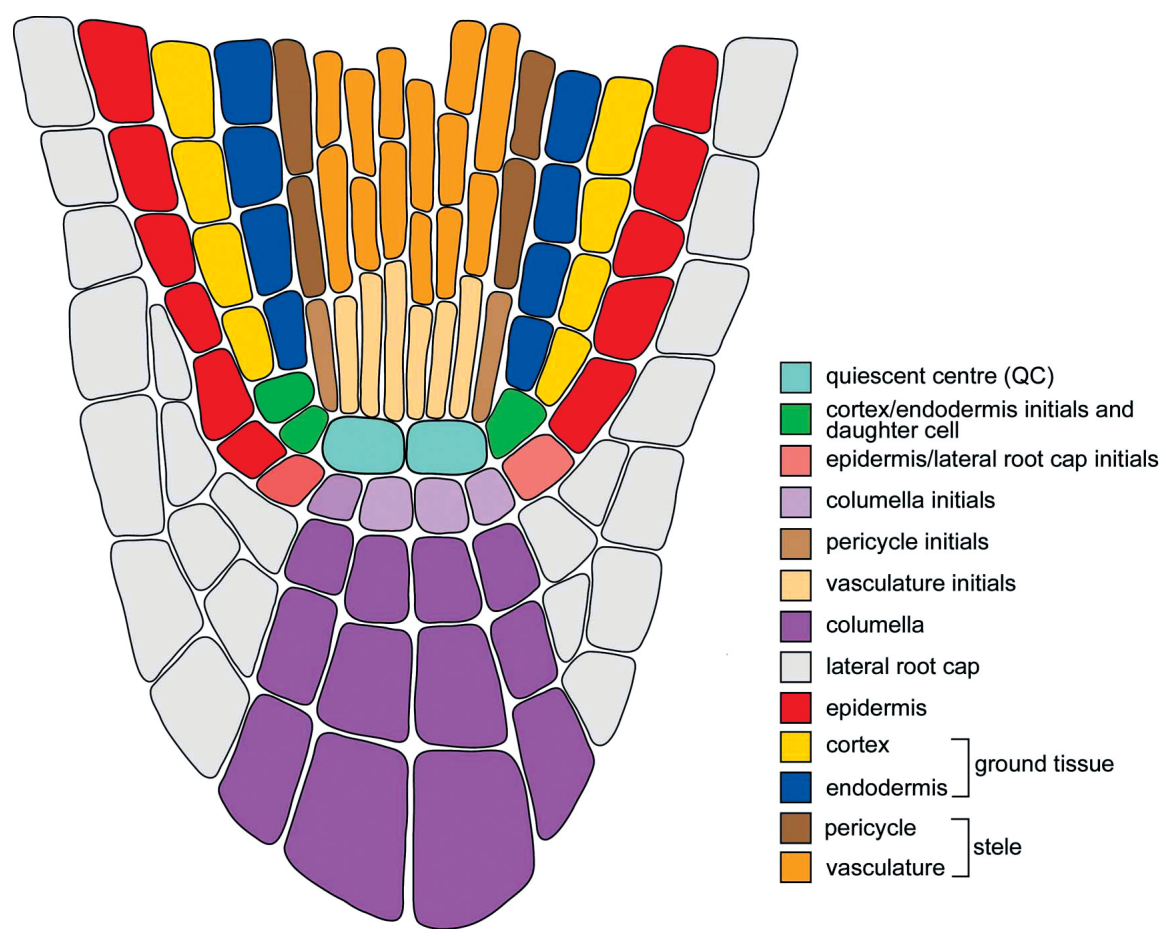

Fig. 5. Organisation of the root meristem. loss of stem cells, requires the combined activities of both systems, since mutations in either the $C L V$ genes or $A G$ result in indeterminacy.

However, to be a floral meristem is not necessarily the end of it all: occasionally, floral meristems may switch back to an indeterminate state and restart as a shoot meristem. In Arabidopsis, this process called floral reversion can be induced by manipulating the flower-inducing photoperiodic stimulus, or by reducing $\angle F Y$ or $A G$ activity. When plants heterozygous for a lfy mutation are cultivated under short-day conditions, flowers can revert even after carpel formation and grow a new shoot from gynoecium cells that express $A G$ (Okamuro et al., 1996). This indicates that even high levels of $A G$ expression in the meristem centre are not sufficient for a permanent repression of stem cell fate. Maintenance of the determinate floral state may require not only downregulation of WUS via $A G$ and the $C L V$ genes, but also repression of other, shoot meristem specific genes. Indeed, it has been proposed that $\angle F Y$ has a dual function in activating flower specific, but repressing shoot specific gene expression (Okamuro et al., 1996, Parcy et al., 2002).

\section{Integration of meristem activity: new genes and mutants}

We have so far just begun to understand how the control of stem cell number is integrated with the overall growth and development of the shoot system and with the formation of organs. Genetic screens for mutants that are affected in meristem maintenance continue to reveal new gene functions that have to be incorporated into the existing regulatory networks. Some of them, like HANABA TARANU (HAN) gene of Arabidopsis, are required to initiate and maintain the precise expression pattern of WUS. $H A N$ encodes a GATA-3-like transcription factor that is expressed at the boundaries between floral whorls and between newly arising organs and the meristem. In han mutants, both WUS and the stem cell marker $C L V 3$ are expressed in more diffuse domains and meristem size decreases. (Zhao et al., 2004). han/c/v double mutants show increased fasciation (cell proliferation in the meristem) compared to $\mathrm{c} / \mathrm{v}$ single mutants, indicating that these gens act in separate pathways to regulate WUS. Interestingly, increased expression of $H A N$ causes growth retardation and severly affects meristem shape and function, indicating that $H A N$ controls cell proliferation and differentiation. HAN expression partially overlaps during early embryogenesis with WUS expression, but not during later shoot and floral meristem development, when HAN RNA is found only in meristem-organ boundaries. At these stages, HAN could act only indirectly to produce a signal that controls either division of WUS -expressing cells, or WUS expression levels in the central meristem domain. This variability of shoot meristem size and activity in han mutant or overexpressing plants suggests that a HAN dependent mechanisms acts in the wildtype, stabilizing meristem size during development. During normal development, the requirements for cell production from the mer- 
istem may change during growth phases and a HAN controlled pathway, acting from the meristem-organ interface, could coordinate meristem size with the rate of organ production.

HAIRY MERISTEM (ham) mutants of petunia fail to maintain a shoot meristem, similar to ter mutants (TER is a homologue of WUS) (Stuurman et al., 2002). ham/ter double mutants display additive effects, indicating that these genes act in parallel pathways. The $H A M$ gene encodes a transcription factor of the GRAS family that is expressed in the provasculature of the stem and developing organs. Other GRAS genes have been shown to be involved in root cell type specification and stem cell maintenance (see below), but also in mediating gibberellic acid signaling. The exact mode of action of $H A M$ is unknown, but its mutant phenotype together with its expression pattern indicates that $H A M$ acts non-cellautonomously in a novel pathway that links stem cell perpetuation in meristems with the generation of differentiated cell types at the periphery.

Similar to ham mutants of petunia, the shoot meristems of Arabidopsis Dornröschen (drn-D) mutants terminate prematurely. The affected gene, DRN/ESR1, encodes an AP2/ERF type transcription factor that is misexpressed in $d r n-D$ mutants, resulting in altered regulation of $C L V 3, W U S, S T M$ and possibly other target genes (Kirch et al., 2003). Interestingly, DRN/ESR1 is normally transcribed in the central zone of shoot and floral meristems and in the anlagen of lateral organs, where expression is maintained for a short period at the tip of the primordium. For example, DRN/ESR1 is expressed in single epidermal cells of the ovule anlagen and remains expressed in the most apical cell of the growing ovule. The common theme underlying the spatial distribution of DRN/ESR1 mRNA may be "apical position" for all meristems and organs. Cellular differentiation of lateral organs starts from the organ tip and may be promoted by $D R N$ expression at this position. DRN expression in the central zone of meristems would then counteract or balance stem cell promoting signals by fostering the exit of stem cells to the TA cell fate.

\section{Deconstructing plant stem cell niches: root mer- istems}

\section{Organisation of the Arabidopsis root}

The Arabidopsis root comprises a rather simple structure of concentrically organised cell layers forming (from the outside to the inside) epidermis, cortex, endodermis, pericycle and vasculature. Cortex and endodermis together are called the ground tissue and vasculature and pericycle jointly form the stele. The overall root structure can be divided into distinct zones, representing its different developmental stages. The meristematic zone at the root tip contains stem cells that generate all the tissues of the root. This is followed by the elongation zone where the cells undergo regulated elongation and finally the differentiation zone where cells acquire their individual fate. In this region, root hairs appear as an indicator of differentiation (Dolan et al., 1993). The stem cells of the root meristem (also called "initials") give rise to all the cell types in each layer by stereotyped divisions, thereby producing clonally related files of cells (Fig. 5). The division of initials can either be merely anticlinal (like in the columella initials, forming only one cell file or layer) or first anticlinal and then periclinal (in all other initials, e.g. endodermis/cortex initials, forming two or more cell layers) (Dolan et al., 1993). After the initial cell divides, one of the two daughter cells remains an initial cell, whereas the other cell becomes a transit-amplifying cell (TA cell) and after a number of divisions differentiates and acquires its specific fate. The initials surround four mitotically inactive cells called the quiescent centre (QC) that act as a stem cell organizer (Dolan et al., 1993). QC and initial cells together constitute the stem cell niche of the Arabidopsis root. The QC is established in the octant stage of embryogenesis from a single cell, the hypophysis (Scheres et al., 1994). After the hypophysis divides, the upper lens-shaped cell gives rise to the QC, whereas the lower daughter cell becomes the central root cap.

\section{Communication in the root stem cell niche}

Root meristem cells show rigid clonal relationships and both QC, initial cells and their descendants can be easily identified by their position. However, similar to the situation in the shoot stem cell system, the fate of a given cell in the root is not permanently fixed, but depends on signals from its neighbours. This was elegantly revealed by laser ablation of individual QC cells or initials in the Arabidopsis root meristem, showing that these cells can be replaced by their neighbours, which then acquire the appropriate identity (van den Berg et al., 1995) (Fig. 4B). Furthermore, differentiated cells (or TA cells?) appear to signal back to their initials and direct their fate. However, when individual QC cells were ablated, only those initial cells in direct contact to the ablated QC cell lost their stem cell character and started to differentiate (or behaved like their daughter cells) (van den Berg et al., 1997). Noteworthy, this initiation of differentiation did not require an intermediate round of cell divisions. This implies that the QC is a source of a short-range, non-cell autonomous signal, which prevents the differentiation of the surrounding initial cells and thereby maintains their stem cell character. The nature of this signal is not yet known.

The role of the initial cells of the root is equivalent to the role of the $C L V 3$-expressing stem cells in the shoot meristem and QC cells have a role similar to that of the $W U S$-expressing $O C$ cells. However, stem cell fate in the shoot is controlled at the level of cell populations (populational asymmetry), whereas root stem cells seem to be controlled individually by a short-range signal from the QC (individual asymmetry).

\section{A CLV-like pathway acting in the root?}

Several observations indicate that a $C L V$-like signaling pathway may also act to control stem cell fate in the root. Overexpression of three different members of the CLE-protein family (CLV3, CLE19 and CLE40) in the root caused a progressive reduction of root meristem activity, leading to growth termination (CasamitjanaMartinez et al., 2003, Hobe et al., 2003). These root defects are not due to a misspecification of the QC or a loss of initials, but to fewer cell divisions (premature differentation) of their immediate descendants (the TA cells), which is indicated by the formation of differentiated cell types such as root hairs close to the meristem tip. Similar to the $C L V$ signaling pathway in the shoot that regulates the activity of the stem cell population, this $C L E$ signaling pathway in the root also controls the size of a cell population, but not stem cell fate which is regulated at the individual cell level in the root (Fig. 4B). To identify components of $C L E$ signaling pathways in the root, Casamitjana-Martinez and colleagues screened for suppressors of $\underline{L} L P 1 / C L E$ (SOL) overexpression 
and found that $C L E$ signaling requires the activity of $S O L 1$. The SOL1 protein resembles $\mathrm{Zn}^{2+}$-carboxypeptidases and could process CLE proteins into an active form. No root specific receptor protein for CLE has been found to date, but in both rice and Arabidopsis, homeobox genes closely related to WUS were shown to be expressed specifically in the QC (Haecker et al., 2004, Kamiya et al., 2003).

\section{Positioning the root stem cell niche}

The root stem cell niche is laid out during early embryogenesis. One of the most important factors that pattern the early embryo is the phytohormone auxin. Auxin is transported in a polar fashion and auxin carrier or response mutants display root patterning defects. In the developing globular embryo, auxin becomes redistributed through the activity of PIN proteins and accumulates in the basal half of the embryo, where root stem cells are specified (the role of auxin in embryogenesis will be discussed in detail in another article in this issue) (Fig. 3). Auxin acts through a class of transcription factors (auxin response factors, or ARF) such as MONOPTEROS (MP) that interact directly with promoter elements of auxin inducible genes. mp mutants fail to produce the hypophysis and do not develop root tissue (Hardtke and Berleth, 1998). The auxin response maximum in the developing root, monitored using a synthetic auxin-responsive promoter driving the GUS reporter gene (Sabatini etal., 1999), is found at the distal root tip. Exogenous application of auxin, or shifting the auxin maximum using transport inhibitors can induce the formation of ectopic QC and stem cells, consistent with auxin positioning the stem cell niche in the root. However, the transcription factors that act downstream of auxin signal transduction were unknown. Aida and colleagues have now shown that auxin inducible expression of the PLETHORA ( PLT1 and 2) genes of Arabidopsis, encoding AP2 type transcription factors, is required for $Q C$ specification and stem cell activity. The expression patterns of PLT1 and 2, first in the basal region of the early embryo and later in the distal root meristem, overlap with the auxin maxima and identify the root stem cell niche. Double mutants of $p / t 1$ and $p / t 2$ suffer from a (not understood) size reduction of cortex cells, but more importantly, they appear to lack a functional QC and show differentiation of stem cells (Aida et al., 2004). But are PLT1 and 2 sufficient to induce stem cell fate? Misexpression of $P L T$ genes induce all cell identities that are derived from the basal part of the embryo, such as hypocotyl and root. Some transgenic seedlings appeared to consist entirely of root cell types, including the shedding and starch-granule containing columella cells of the distal root tip. Most importantly, QC marker genes were activated at new positions without a prior accumulation of auxin at these sites, suggesting that once activated by auxin response factors like MP, the $P L T$ genes can act independently to establish root meristem cell fates.

Besides the PLT pathway, two members of the GRAS gene family, SHORTROOT (SHR) and SCARECROW (SCR), are required for $Q C$ specification and stem cell sustainment in the root. SHR RNA is made in the provascular tissue, but SHR protein moves out to the adjacent cells (endodermis and $Q C$ ) where it enters the nucleus to activate $S C R$ transcription. SCR in turn appears to inhibit SHR from moving further outwards to adjacent layers (Heidstra et al., 2004). SCR expression in the QC is required for $\mathrm{QC}$ identity, so $S C R$ can act only cell-autonomously. However, expression of $S C R$ in the $Q C$ region could not rescue the root meristem defects of shr mutant seedlings, indicating that the role of $S H R$ is not limited to $S C R$ activation and that presence of both SHR and SCR protein in the QC is required to maintain it (Sabatini et al., 2003). Both proteins are also expressed in the entire endodermal cell layer (Helariutta et al., 2000, Nakajima et al., 2001) and exogenous auxin can transform these cells into QC with adjacent stem cells.

From these data, Aida and colleagues have proposed a model for patterning the root stem cell niche: First, auxin accumulation at the basal end of the embryo activates ARFs such as MP, which upregulate PLT expression. Then, SHR accumulates in provascular cells and promotes $S C R$ expression in the adjacent cell layer and the combined activities of PLT, SCR and SHR specify the $\mathrm{QC}$ cells. Finally, cells surrounding the $\mathrm{QC}$ that also express PLT (but not SCR or SHR) acquire stem cell identity in response to (so far unknown) short range signals from the QC (Aida et al., 2004).

\section{Acknowledgement}

Work in the Simon laboratory is funded by the DFG.

\section{References}

AIDA, M., BEIS, D., HEIDSTRA, R., WILLEMSEN, V., BLILOU, I., GALINHA, C., NUSSAUME, L., NOH, Y.S., AMASINO, R. and SCHERES, B. (2004). The PLETHORA Genes Mediate Patterning of the Arabidopsis Root Stem Cell Niche. Cell 119: 109-20.

BLAU, H.M., BRAZELTON, T.R. and WEIMANN, J.M. (2001). The evolving concept of a stem cell: entity or function? Cell 105: 829-41.

BRAND, U., FLETCHER, J.C., HOBE, M., MEYEROWITZ, E.M. and SIMON, R. (2000). Dependence of stem cell fate in Arabidopsis on a feedback loop regulated by CLV3 activity. Science 289: 617-9.

BRAND, U., GRÜNEWALD, M., HOBE, M. and SIMON, R. (2002). Regulation of CLV3 expression by two homeobox genes in Arabidopsis. Plant Physiol 129: 565-75.

BUSCH, M.A., BOMBLIES, K. and WEIGEL, D. (1999). Activation of a floral homeotic gene in Arabidopsis. Science 285: 585-7.

CASAMITJANA-MARTINEZ, E., HOFHUIS, H.F., XU, J., LIU, C.M., HEIDSTRA, R. and SCHERES, B. (2003). Root-specific CLE19 overexpression and the sol1/ 2 suppressors implicate a CLV-like pathway in the control of Arabidopsis root meristem maintenance. Current Biology 13: 1435-1441.

CHEN, X., LIU, J., CHENG, Y. and JIA, D. (2002). HEN1 functions pleiotropically in Arabidopsis development and acts in $\mathrm{C}$ function in the flower. Development 129: 1085-94.

CHEN, X. and MEYEROWITZ, E.M. (1999). HUA1 and HUA2 are two members of the floral homeotic AGAMOUS pathway. Mol Cell 3: 349-60.

CHENG, Y., KATO, N., WANG, W., LI, J. and CHEN, X. (2003). Two RNA binding proteins, HEN4 and HUA1, act in the processing of AGAMOUS pre-mRNA in Arabidopsis thaliana. Dev Cel/ 4: 53-66.

CLARK, S.E., WILLIAMS, R.W. and MEYEROWITZ, E.M. (1997). The CLAVATA1 gene encodes a putative receptor kinase that controls shoot and floral meristem size in Arabidopsis. Cell 89: 575-85.

COCK, J.M. and MCCORMICK, S. (2001). A large family of genes that share homology with CLAVATA3. Plant Physiol 126: 939-42.

DIEVART, A., DALAL, M., TAX, F.E., LACEY, A.D., HUTTLY, A., LI, J. and CLARK, S.E. (2003). CLAVATA1 Dominant-Negative Alleles Reveal Functional Overlap between Multiple Receptor Kinases That Regulate Meristem and Organ Development. Plant Cel/ 15: 1198-1211.

DOLAN, L., JANMAAT, K., WILLEMSEN, V., LINSTEAD, P., POETHIG, S., ROBERTS, K. and SCHERES, B. (1993). Cellular organisation of the Arabidopsis thaliana root. Development 119: 71-84.

FLETCHER, J.C., BRAND, U., RUNNING, M.P., SIMON, R. and MEYEROWITZ, E.M. (1999). Signaling of cell fate decisions by CLAVATA3 in Arabidopsis shoot 
meristems. Science 283: 1911-4.

GALLOIS, J.L., NORA, F.R., MIZUKAMI, Y. and SABLOWSKI, R. (2004). WUSCHEL induces shoot stem cell activity and developmental plasticity in the root meristem. Genes Dev 18: 375-80.

GROSS-HARDT, R., LENHARD, M. and LAUX, T. (2002). WUSCHEL signaling functions in interregional communication during Arabidopsis ovule development. Genes Dev 16: 1129-38.

HAECKER, A., GROSS-HARDT, R., GEIGES, B., SARKAR, A., BREUNINGER, H., HERRMANN, M. and LAUX, T. (2004). Expression dynamics of WOX genes mark cell fate decisions during early embryonic patterning in Arabidopsis thaliana. Development 131: 657-68.

HARDTKE, C.S. and BERLETH, T. (1998). The Arabidopsis gene MONOPTEROS encodes a transcription factor mediating embryo axis formation and vascular development. EMBO J 17: 1405-11.

HECHT, V., VIELLE-CALZADA, J.P., HARTOG, M.V., SCHMIDT, E.D., BOUTILIER, K., GROSSNIKLAUS, U. and DE VRIES, S.C. (2001). The Arabidopsis SOMATIC EMBRYOGENESIS RECEPTOR KINASE 1 gene is expressed in developing ovules and embryos and enhances embryogenic competence in culture. Plant Physiol 127: 803-16.

HEIDSTRA, R., WELCH, D. and SCHERES, B. (2004). Mosaic analyses using marked activation and deletion clones dissect Arabidopsis SCARECROW action in asymmetric cell division. Genes Dev 18: 1964-9.

HELARIUTTA, Y., FUKAKI, H., WYSOCKA-DILLER, J., NAKAJIMA, K., JUNG, J., SENA, G., HAUSER, M.T. and BENFEY, P.N. (2000). The SHORT-ROOT gene controls radial patterning of the Arabidopsis root through radial signaling. Cell 101: 555-67.

HOBE, M., MÜLLER, R., GRÜNEWALD, M., BRAND, U. and SIMON, R. (2003). Loss of CLE40, a protein functionally equivalent to the stem cell restricting signal CLV3, enhances root waving in Arabidopsis. Dev Genes Evol 213: 371381.

ISHIGURO, S., WATANABE, Y., ITO, N., NONAKA, H., TAKEDA, N., SAKAI, T., KANAYA, H. and OKADA, K. (2002). SHEPHERD is the Arabidopsis GRP94 responsible for the formation of functional CLAVATA proteins. EMBO J. 21: 898-908.

JEONG, S. and CLARK, S.E. (2004). Photoperiod regulates flower meristem development in Arabidopsis thaliana. Genetics.

JEONG, S., TROTOCHAUD, A.E. and CLARK, S.E. (1999). The Arabidopsis CLAVATA2 gene encodes a receptor-like protein required for the stability of the CLAVATA1 receptor-like kinase. Plant Cell 11: 1925-34.

KAMIYA, N., NAGASAKI, H., MORIKAMI, A., SATO, Y. and MATSUOKA, M. (2003). Isolation and characterization of a rice WUSCHEL-type homeobox gene that is specifically expressed in the central cells of a quiescent center in the root apical meristem. Plant $J$ 35: 429-41.

KAYES, J.M. and CLARK, S.E. (1998). CLAVATA2, a regulator of meristem and organ development in Arabidopsis. Development 125: 3843-51.

KIRCH, T., SIMON, R., GRÜNEWALD, M. and WERR, W. (2003). The DORNRÖSCHEN/ENHANCER OF SHOOT REGENERATION1 Gene of Arabidopsis Acts in the Control of Meristem Cell Fate and Lateral Organ Development. Plant Cell 15: 694-705.

LAUX, T., MAYER, K.F., BERGER, J. and JURGENS, G. (1996). The WUSCHEL gene is required for shoot and floral meristem integrity in Arabidopsis. Development 122: 87-96.

LENHARD, M., BOHNERT, A., JURGENS, G. and LAUX, T. (2001). Termination of stem cell maintenance in Arabidopsis floral meristems by interactions between WUSCHEL and AGAMOUS. Cel/ 105: 805-14.

LENHARD, M., JURGENS, G. and LAUX, T. (2002). The WUSCHEL and SHOOTMERISTEMLESS genes fulfil complementary roles in Arabidopsis shoot meristem regulation. Development 129: 3195-206.

LI, J., JIA, D. and CHEN, X. (2001). HUA1, a regulator of stamen and carpel identities in Arabidopsis, codes for a nuclear RNA binding protein. Plant Cel/ 13: 2269-81.

LOHMANN, J.U., HONG, R.L., HOBE, M., BUSCH, M.A., PARCY, F., SIMON, R. and WEIGEL, D. (2001). A molecular link between stem cell regulation and floral patterning in Arabidopsis. Cell 105: 793-803.

MATSUMOTO, N. and OKADA, K. (2001). A homeobox gene, PRESSED FLOWER, regulates lateral axis-dependent development of Arabidopsis flowers. Genes Dev 15: 3355-64.

MAYER, K.F., SCHOOF, H., HAECKER, A., LENHARD, M., JURGENS, G. and LAUX, T. (1998). Role of WUSCHEL in regulating stem cell fate in the Arabidopsis shoot meristem. Cell 95: 805-15.

MIZUKAMI, Y. and MA, H. (1992). Ectopic expression of the floral homeotic gene AGAMOUS in transgenic Arabidopsis plants alters floral organ identity. Cel/ 71 : 119-31.

NAKAJIMA, K., SENA, G., NAWY, T. and BENFEY, P.N. (2001). Intercellular movement of the putative transcription factor SHR in root patterning. Nature 413: 307-11.

OKAMURO, J.K., DEN BOER, B.G., LOTYS-PRASS, C., SZETO, W. and JOFUKU, K.D. (1996). Flowers into shoots: photo and hormonal control of a meristem identity switch in Arabidopsis. Proc Natl Acad Sci USA 93: 13831-6.

PARCY, F., BOMBLIES, K. and WEIGEL, D. (2002). Interaction of LEAFY, AGAMOUS and TERMINAL FLOWER1 in maintaining floral meristem identity in Arabidopsis. Development 129: 2519-27.

PARCY, F., NILSSON, O., BUSCH, M.A., LEE, I. and WEIGEL, D. (1998). A genetic framework for floral patterning. Nature 395: 561-6.

REDDY, G.V., HEISLER, M.G., EHRHARDT, D.W. and MEYEROWITZ, E.M (2004). Real-time lineage analysis reveals oriented cell divisions associated with morphogenesis at the shoot apex of Arabidopsis thaliana. Development 131: 4225-37.

REINHARDT, D., FRENZ, M., MANDEL, T. and KUHLEMEIER, C. (2003). Microsurgical and laser ablation analysis of interactions between the zones and layers of the tomato shoot apical meristem. Development 130: 4073 - 83.

ROJO, E., SHARMA, V.K., KOVALEVA, V., RAIKHEL, N.V. and FLETCHER, J.C. (2002). CLV3 is localized to the extracellular space, where it activates the Arabidopsis CLAVATA stem cell signaling pathway. Plant Cell 14: 969-77.

SABATINI, S., BEIS, D., WOLKENFELT, H., MURFETT, J., GUILFOYLE, T., MALAMY, J., BENFEY, P., LEYSER, O., BECHTOLD, N., WEISBEEK, P. et al. (1999). An auxin-dependent distal organizer of pattern and polarity in the Arabidopsis root. Cel/ 99: 463-72.

SABATINI, S., HEIDSTRA, R., WILDWATER, M. and SCHERES, B. (2003). SCARECROW is involved in positioning the stem cell niche in the Arabidopsis root meristem. Genes Dev 17: 354-8.

SCHERES, B., WOLKENFELT, H., WILLEMSEN, V., TERLOUW, M., LAWSON, E., DEAN, C. and WEISBEEK, P. (1994). Embryonic origin of the Arabidopsis primary root and root meristem initials. Development 120: 2475-2487.

SCHOOF, H., LENHARD, M., HAECKER, A., MAYER, K.F., JURGENS, G. and LAUX, T. (2000). The stem cell population of Arabidopsis shoot meristems in maintained by a regulatory loop between the CLAVATA and WUSCHEL genes. Cell 100: 635-44.

SHARMA, V.K., RAMIREZ, J. and FLETCHER, J.C. (2003). The Arabidopsis CLV3like (CLE) genes are expressed in diverse tissues and encode secreted proteins. Plant Mol Biol 51: 415-25.

SIEBURTH, L.E. and MEYEROWITZ, E.M. (1997). Molecular dissection of the AGAMOUS control region shows that cis elements for spatial regulation are located intragenically. Plant Cell 9: 355-65.

STEWART, R.N. and DERMEN, H. (1970). Determination of number and mitotic activity of shoot apical initial cells by analysis of mericlinal chimeras. Am. J. Botany 57: 816-826.

STONE, J.M., TROTOCHAUD, A.E., WALKER, J.C. and CLARK, S.E. (1998). Control of meristem development by CLAVATA1 receptor kinase and kinaseassociated protein phosphatase interactions. Plant Physiol 117: 1217-25.

STUURMAN, J., JAGGI, F. and KUHLEMEIER, C. (2002). Shoot meristem maintenance is controlled by a GRAS-gene mediated signal from differentiating cells. Genes Dev 16: 2213-8.

SUZAKI, T., SATO, M., ASHIKARI, M., MIYOSHI, M., NAGATO, Y. and HIRANO, H.Y. (2004). The gene FLORAL ORGAN NUMBER1 regulates floral meristem size in rice and encodes a leucine-rich repeat receptor kinase orthologous to Arabidopsis CLAVATA1. Development 131: 5649-57.

SZYMKOWIAK, E.J. and SUSSEX, I.M. (1992). The internal meristem layer (L3) determines floral meristem size and carpel number in tomato periclinal chimeras. Plant Cell 4: 1089-100. 
TAGUCHI-SHIOBARA, F., YUAN, Z., HAKE, S. and JACKSON, D. (2001). The fasciated ear2 gene encodes a leucine-rich repeat receptor-like protein that regulates shoot meristem proliferation in maize. Genes Dev 15: 2755-66.

TROTOCHAUD, A.E., HAO, T., WU, G., YANG, Z. and CLARK, S.E. (1999). The CLAVATA1 receptor-like kinase requires CLAVATA3 for its assembly into a signaling complex that includes KAPP and a Rho-related protein. Plant Cel/ 11: 393-406.

VAN DEN BERG, C., WILLEMSEN, V., HAGE, W., WEISBEEK, P. and SCHERES, B. (1995). Cell fate in the Arabidopsis root meristem determined by directional signalling. Nature 378: $62-5$.

VAN DEN BERG, C., WILLEMSEN, V., HENDRIKS, G., WEISBEEK, P. and SCHERES, B. (1997). Short-range control of cell differentiation in the Arabidopsis root meristem. Nature 390: 287-9.

WANG, W. and CHEN, X. (2004). HUA ENHANCER3 reveals a role for a cyclindependent protein kinase in the specification of floral organ identity in Arabidopsis. Development 131: 3147-56.

WATT, F.M. and HOGAN, B.L. (2000). Out of Eden: stem cells and their niches. Science 287: 1427-30.

WEIGEL, D., ALVAREZ, J., SMYTH, D.R., YANOFSKY, M.F. and MEYEROWITZ, E.M. (1992). LEAFY controls floral meristem identity in Arabidopsis. Cel/ 69: 843-59.
WESTERN, T.L., CHENG, Y., LIU, J. and CHEN, X. (2002). HUA ENHANCER2, a putative DExH-box RNA helicase, maintains homeotic $B$ and $C$ gene expression in Arabidopsis. Development 129: 1569-81.

WILLIAMS, R.W., WILSON, J.M. and MEYEROWITZ, E.M. (1997). A possible role for kinase-associated protein phosphatase in the Arabidopsis CLAVATA1 signaling pathway. Proc Natl Acad Sci USA 94: 10467-72.

YANOFSKY, M.F., MA, H., BOWMAN, J.L., DREWS, G.N., FELDMANN, K.A. and MEYEROWITZ, E.M. (1990). The protein encoded by the Arabidopsis homeotic gene agamous resembles transcription factors. Nature 346: 35-9.

YU, L.P., MILLER, A.K. and CLARK, S.E. (2003). POLTERGEIST Encodes a Protein Phosphatase $2 \mathrm{C}$ that Regulates CLAVATA Pathways Controlling Stem Cell Identity at Arabidopsis Shoot and Flower Meristems. Curr Biol 13: 179-88.

YU, L.P., SIMON, E.J., TROTOCHAUD, A.E. and CLARK, S.E. (2000). POLTERGEIST functions to regulate meristem development downstream of the CLAVATA loci. Development 127: 1661-70.

ZHAO, Y., MEDRANO, L., OHASHI, K., FLETCHER, J.C., YU, H., SAKAI, H. and MEYEROWITZ, E.M. (2004). HANABA TARANU Is a GATA Transcription Factor That Regulates Shoot Apical Meristem and Flower Development in Arabidopsis. Plant Cell.

ZUO, J., NIU, Q.W., FRUGIS, G. and CHUA, N.H. (2002). The WUSCHEL gene promotes vegetative-to-embryonic transition in Arabidopsis. Plant J 30: 349-59. 